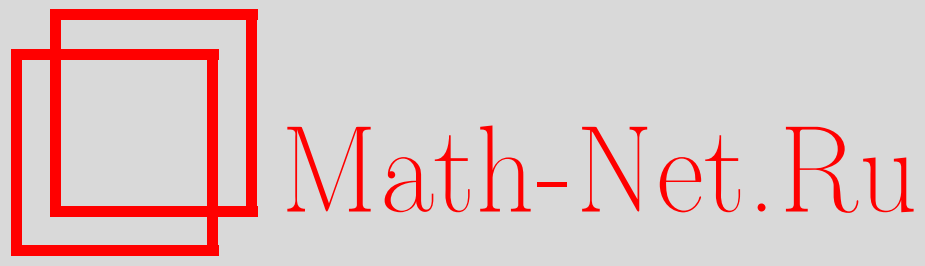

А. Г. Хованский, О монодромии многозначной функции на ее множестве ветвления, Функи. анализ и его прил., 2003, том 37, выпуск 2, 65-74

DOI: https://doi.org/10.4213/faa149

Использование Общероссийского математического портала Math-Net.Ru подразумевает, что вы прочитали и согласны с пользовательским соглашением

http://www.mathnet.ru/rus/agreement

Параметры загрузки:

IP : 3.91 .87 .62

26 апреля 2023 г., 08:46:45

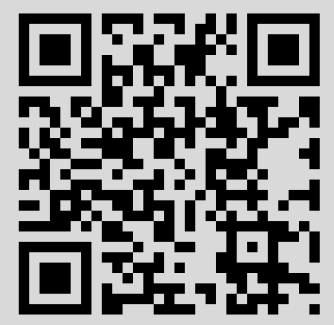


2003, т. 37, вып. 2, с. 65-74

УДК 517.9

\title{
О монодромии многозначной функции на ее множестве ветвления*
}

\author{
(c) 2003. А. Г. ХОВАНСКИЙ
}

Многозначная аналитическая функция в $\mathbb{C}^{n}$ называется $\mathcal{S}$-функцией, если множество ее особых точек покрывается счетным числом аналитических подмножеств (и занимает, следовательно, очень малую часть пространства $\mathbb{C}^{n}$ ). При отображении $\pi:\left(Y, y_{0}\right) \rightarrow\left(\mathbb{C}^{n}, a\right)$ топологического пространства $Y$ в $\mathbb{C}^{n}$ росток $f_{a} \mathcal{S}$-функции $f$ в точке $a$ может индуцировать многозначную функцию на пространстве $Y$. Для этого нужно, чтобы росток $f_{a}$ аналитически продолжался вдоль образа любой кривой из пространства $Y$, начинающейся в отмеченной точке $y_{0}$. Такая ситуация возможна, даже если точка $a$ лежит в множестве особых точек функции $f$ (некоторые из ростков многозначной функции $f$ могут оказаться неособыми в особых точках этой функции) и пространство $Y$ отображается в это множество.

Можно ли оценить группы монодромии индуцированных таким способом многозначных функций через группу монодромии исходной $\mathcal{S}$-функции $f$ (верно ли, например, что если группа монодромии $\mathcal{S}$-функции $f$ разрешима, то разрешима и группа монодромии каждой функции, индуцированной $\mathcal{S}$-функцией $f)$ ? В настоящей статье получен положительный ответ на этот вопрос (см. $\$ 4,5)$.

Это совсем не очевидно, если множество особых точек функции $f$ незамкнуто. Отметим, кстати, что $\mathcal{S}$-функции с незамкнутыми множествами особых точек не являются чем-то необычным. Именно такими, как правило, бывают многозначные элементарные функции. Рассмотрим, например, функцию $\varphi$ одной переменной, определенную формулой

$$
\varphi(u)=\ln \sum_{j=1}^{k} \lambda_{j} \ln \left(u-a_{j}\right),
$$

в которой $a_{j}, j=1, \ldots, k,-$ различные комплексные числа. Если подгруппа $\Lambda$ аддитивной группы комплексных чисел, порожденная коэффициентами $\lambda_{1}, \ldots, \lambda_{k}$, всюду плотна на комплексной прямой, то функция $\varphi$ имеет всюду плотное множество особых точек и континуальную группу монодромии. Если число слагаемых $k$ в формуле для функции $\varphi$ больше двух, то группа $\Lambda$, как правило, будет всюду плотной в $\mathbb{C}$.

Описание связи группы монодромии исходной $\mathcal{S}$-функции с группами монодромии индуцированных таким способом функций потребовало введения операции индуцированного замыкания групп (см. §2). В свою очередь, использование этой операции вынуждает пересмотреть определения различных классов пар групп

*Работа выполнена при частичной поддержке Канадского гранта OGP0156833.

3 Функциональный анализ и его приложения, т. 37, вып. 2 
(см. §5), встречающихся в одномерном топологическом варианте теории Галуа [1-3]. Настоящая статья - шаг к построению многомерного варианта этой теории $[4,5]$, развивающей топологический подход Абеля и Арнольда к проблемам неразрешимости (по поводу этого подхода см. [6-8]). В статье строятся определения, позволяющие работать с функциями многих переменных, имеющими всюду плотные множества особых точек и континуальные группы монодромии.

Пользуюсь случаем поблагодарить В. И. Арнольда, который был моим научным руководителем во время работы над диссертацией [2]. Я признателен моей жене Т. В. Белокриницкой за большую помощь при написании этой статьи.

\section{§1. $\mathcal{S}$-функции}

В одномерном топологическом варианте теории Галуа центральную роль играют $\mathcal{S}$-функции, т. е. многозначные аналитические функции одной переменной, множество особых точек которых не более чем счетно. Обобщим понятие класса $\mathcal{S}$-функций на многомерный случай. Все утверждения этого параграфа несложны и доказываются точно так же, как их одномерные аналоги $[2,3]$; поэтому мы не будем останавливаться на их доказательстве.

Подмножество $A$ в связном $k$-мерном аналитическом многообразии $\mathbf{M}$ назовем тощим, если существует счетное множество открытых областей $U_{i} \subset \mathbf{M}$ и счетное множество собственных аналитических подмножеств $A_{i} \subset U_{i}$ в этих областях, таких, что $A \subseteq \bigcup A_{i}$. Многозначную аналитическую функцию на многообразии $\mathbf{M}$ назовем $\mathcal{S}$-функцией, если множество ее особых точек является тощим. Уточним это определение.

Два регулярных ростка $f_{a}$ и $g_{b}$, заданных в точках $a$ и $b$ многообразия $\mathbf{M}$, называются эквивалентными, если росток $g_{b}$ получается из ростка $f_{a}$ регулярным продолжением вдоль некоторой кривой. Каждый росток $g_{b}$, эквивалентный ростку $f_{a}$, называется также ростком многозначной аналитической функции $f$, порожденной ростком $f_{a}$. Точка $b \in \mathbf{M}$ называется особой для ростка $f_{a}$, если существует кривая $\gamma:[0,1] \rightarrow \mathbf{M}, \gamma(0)=a, \gamma(1)=b$, такая, что росток $f_{a}$ не может быть регулярно продолжен вдоль этой кривой, но для любого $t, 0 \leqslant t<1$, регулярно продолжается вдоль укороченной кривой $\gamma:[0, t] \rightarrow \mathbf{M}$. Легко видеть, что у эквивалентных ростков множества особых точек совпадают. Росток называется $\mathcal{S}$-ростком, если множество его особых точек является тощим. Многозначная аналитическая функция называется $\mathcal{S}$-функцией, если каждый ее росток является $\mathcal{S}$-ростком.

Фиксируем произвольную риманову метрику $\rho$ на многообразии $\mathbf{M}$.

ЛЕМмА О СНЯТИИ КРИВОЙ С ТОЩЕГО мНОЖеСтвА. Пусть А- тощее множество на многообразии $\mathbf{M}, \gamma:[0,1] \rightarrow \mathbf{M}-$ кривая и $\varphi-$ непрерывная положительная функция на интервале $0<t<1$. Тогда существует кривая $\widehat{\gamma}:[0,1] \rightarrow \mathbf{M}$, такая, ито $\widehat{\gamma}(t) \notin A$ при $0<t<1$ и $\rho(\gamma(t), \widehat{\gamma}(t))<\varphi(t)$.

Кроме множества особых точек, удобно рассматривать и другие множества, вне которых функция неограниченно продолжается. Тощее множество $A$ называется запрещенным множеством для регулярного ростка $f_{a}$, если росток $f_{a}$ регулярно продолжается вдоль кривой $\gamma(t), \gamma(0)=a$, пересекающей множество $A$ лишь, может быть, в начальный момент.

ТЕОРЕМА О ЗАПРЕЩЕННОМ МНОЖЕСТВЕ. Тощее множество является запрещенным множеством ростка, если и только если оно содержит множество 
его особых точек. В частности, росток обладает некоторым запрещенным множеством, если и только если он является ростком $\mathcal{S}$-функциии.

Группа монодромии $\mathcal{S}$-функции $f$ с запрещенным множеством $A$ (или, короче, группа $A$-монодромии) - это группа всех перестановок листов функции $f$, которые происходят при обходе точек множества $A$. Остановимся на этом подробнее.

Пусть $X$ - множество всех ростков $\mathcal{S}$-функции $f$ в точке $a$, не лежащей в множестве $A$. Возьмем замкнутую кривую $\gamma$ в $\mathbf{M} \backslash A$ с началом в точке $a$. Продолжение каждого ростка из множества $X$ вдоль кривой $\gamma$ приводит к ростку из множества $X$. Итак, каждой замкнутой кривой $\gamma$ соответствует отображение множества $X$ в себя, причем гомотопным в $\mathbf{M} \backslash A$ кривым отвечают одинаковые отображения. Произведению кривых отвечает произведение отображений. Возникает гомоморфизм $\tau$ фундаментальной группы множества $\mathbf{M} \backslash A$ в группу $S(X)$ взаимно однозначных преобразований множества $X$. (Здесь и во всей статье мы имеем в виду следующую групповую структуру в группе $S(X)$ : если $f, g-$ взаимно однозначные преобразования множества $X$, то их произведением $f g$ в группе $S(X)$ называется композиция $g \circ f$ отображений $f$ и $g$. .) Группой $A$-монодромии $\mathcal{S}$-функции $f$ называется образ фундаментальной группы $\pi_{1}(\mathbf{M} \backslash A, a)$ в группе $S(X)$ при гомоморфизме $\tau$.

Группа $A$-монодромии - это не только абстрактная группа, но и группа транзитивных перестановок листов функции (транзитивность этой группы легко выводится из леммы о снятии кривой с тощего множества). Алгебраически такой объект задается парой групп: группой перестановок и ее подгруппой, являющейся стационарной подгруппой некоторого элемента.

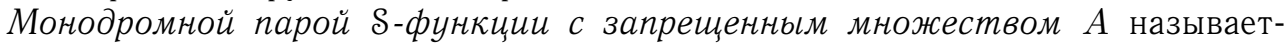
ся пара групп, состоящая из группы $A$-монодромии и стационарной подгруппы некоторого листа этой функции. Эта пара групп определена корректно, т. е. с точностью до изоморфизма не зависит ни от выбора точки $a$, ни от выбора листа функции. В случае, когда запрещенное множество $A$ совпадает с множеством особых точек функции, упоминание о запрещенном множестве мы будем опускать и говорить о группе монодромии и монодромной паре этой функции. В случае, когда множество особых точек функции незамкнуто, группа $A$-монодромии может оказаться собственной подгруппой группы монодромии этой функции.

Группа $S(X)$ наделена топологией (см. §3).

ПредложЕНИЕ. Замыкание в группе $S(X)$ группь $A$-монодромии $\mathcal{S}$-функциии $f$ не зависит от выбора запрещенного множества $A$ и содержит группу монодромии функции $f$. При этом замыкание стационарной подгруппь фиксированного листа $f_{a}$ относительно действия группь A-монодромии содержит стациионарную подгруппу этого листа относительно действия группь монодромии.

\section{§2. Почти гомоморфизмы и индуцированные замыкания}

Нам понадобится конструкция, сопоставляющая каждой группе преобразований множества $X$ некоторую группу преобразований его подмножества $L$ (см. §3). Для изучения ее свойств удобно воспользоваться понятиями почти гомоморфизма и индуцированного замыкания.

Пусть $T$ - топологическое пространство и $S$ - группа, лежащая в $T$.

ОПрЕдЕЛЕНИЕ. Отображение $J: G \rightarrow T$ группы $G$ в пространство $T$ называется почти гомоморфизмом около группь $S$, если: 
1) отображение $J$ переводит единицу группы $G$ в единицу группы $S$;

2 ) для любой точки $a$ группы $S$ и всякой окрестности $V$ в пространстве $T$ точки $a^{-1}$ существует такая окрестность $U$ в пространстве $T$ точки $a$, что для всякой точки $\tilde{a}$ группы $G$, для которой $J(\tilde{a}) \in U$, справедливо включение $J\left(\tilde{a}^{-1}\right) \in V$;

$3)$ для любых точек $a, b$ группы $S$ и любой окрестности $V$ в пространстве $T$ точки $a b$ существуют такие окрестности $U_{1}$ и $U_{2}$ в пространстве $T$ точек $a$ и $b$, что для любых точек $\tilde{a}, \tilde{b}$ группы $G$, для которых $J(\tilde{a}) \in U_{1}$ и $J(\tilde{b}) \in U_{2}$, справедливо включение $J(\tilde{a} \tilde{b}) \in V$.

ОПредЕЛЕНИЕ. Индуизированным замыканием $\bar{G}(S)$ группы $G$ в группе $S$ относительно почти гомоморфизма $J: G \rightarrow T$ около группы $S$ называется пересечение группы $S$ с замыканием $\bar{J}(G)$ в пространстве $T$ образа группы $G$ при отображении $J$.

Опишем свойства индуцированного замыкания. Прежде всего, индуцированное замыкание $\bar{G}(S)$ является подгруппой в группе $S$. Далее, ограничение $J: G_{1} \rightarrow T$ почти гомоморфизма $J: G \rightarrow T$ группь $G$ около группь $S$ на подгруппу $G_{1}$ группь $G$, очевидно, является почти гомоморфизмом группь $G_{1}$ около групnы $S$. Поэтому индуцированное замыкание определено для всех подгрупп группы $G$ одновременно.

Пусть $A_{1}, \ldots, A_{n}$ - алфавит, содержащий $n$ символов. Словом $W$ в этом алфавите называется любое выражение вида $W=A_{i_{1}}^{k_{1}} \ldots A_{i_{N}}^{k_{N}}$, где индексы $i_{1}, \ldots, i_{N}$ принимают любые целые значения от 1 до $n$, а степени $k_{1}, \ldots, k_{N}$ равны плюс или минус единице. Число $k=\left|k_{1}\right|+\cdots+\left|k_{N}\right|$ называется длиной слова $W$. Для всякой группы П и всякой последовательности $a_{1}, \ldots, a_{n}$ ее точек определено значение $W\left(a_{1}, \ldots, a_{n}\right)$ слова $W$, являющееся точкой $a_{i_{1}}^{k_{1}} \ldots a_{i_{n}}^{k_{n}}$ группы П.

ПрЕДЛОЖЕНИЕ. Пусть $J: G \rightarrow T-$ почти гомоморфизм группь $G$ около группь $S$. Тогда для всякого слова $W$, всякого набора точек $a_{1}, \ldots, a_{n}$ групnьь $S$ и всякой окрестности $V$ в пространстве $T$ точки $W\left(a_{1}, \ldots, a_{n}\right) \in S c y$ ществуют такие окрестности $U_{1}, \ldots, U_{n}$ в пространстве $T$ точек $a_{1}, \ldots, a_{n}$, что для всяких точек $\tilde{a}_{1}, \ldots, \tilde{a}_{n}$, для которых $J\left(\tilde{a}_{1}\right) \in U_{1}, \ldots, J\left(\tilde{a}_{n}\right) \in U_{n}$, справедливо включение $J\left(W\left(\tilde{a}_{1}, \ldots, \tilde{a}_{n}\right)\right) \in V$.

ДокАЗАТЕЛЬСтво. Проведем индукцию по длине $k$ слова $W$. Для единственного нетривиального слова $W=A_{i_{1}}^{-1}$ длины один утверждение справедливо по определению почти гомоморфизма. Каждое слово $W$ длины $k>1$ записывается либо в виде $A_{i_{1}} W_{1}$, либо в виде $A_{i_{1}}^{-1} W_{1}$, где $W_{1}-$ слово длины $k-1$. В любом из этих двух случаев по определению почти гомоморфизма для каждой окрестности $V$ точки $W\left(a_{1}, \ldots, a_{n}\right)$ существуют окрестности $V_{1}$ и $V_{2}$ точек $a_{i_{1}}$ и $W_{1}\left(a_{1}, \ldots, a_{n}\right)$, такие, что если точки $\tilde{a}_{1}, \ldots, \tilde{a}_{n}$ группы $G$ удовлетворяют включениям $J\left(\tilde{a}_{i_{1}}\right) \in V_{1}$ и $J\left(W_{1}\left(\tilde{a}_{1}, \ldots, \tilde{a}_{n}\right)\right) \in V_{2}$, то $J\left(W\left(\tilde{a}_{1}, \ldots, \tilde{a}_{n}\right)\right) \in V$. По предположению индукции найдутся такие окрестности $U_{1}, \ldots, U_{n}$ точек $a_{1}, \ldots, a_{n}$, что если элементы $\tilde{a}_{1}, \ldots, \tilde{a}_{n}$ удовлетворяют включениям $J\left(\tilde{a}_{1}\right) \in U_{1}, \ldots, J\left(\tilde{a}_{n}\right) \in$ $U_{n}$, то $J\left(W\left(\tilde{a}_{1}, \ldots, \tilde{a}_{n}\right)\right) \in V_{2}$. Поэтому если точка $\tilde{a}_{i_{1}}$ удовлетворяет включению $J\left(\tilde{a}_{i_{1}}\right) \in U_{i_{1}} \cap V_{1}$, а точки $\tilde{a}_{j}$ при $j \neq i_{1}$ удовлетворяют включениям $J\left(\tilde{a}_{j}\right) \in U_{j}$, то $J\left(W\left(\tilde{a}_{1}, \ldots, \tilde{a}_{n}\right)\right) \in V$. Утверждение доказано. 
Теорема 1. Для всякого почти гомоморфизма $J: G \rightarrow T$ группы $G$ около группь $S$ и всякого нормального делителя $G_{1}$ группь $G$, для которого факторгруппа $G / G_{1}$ коммутативна, индуцированные замыкания $\bar{G}_{1}(S), \bar{G}(S)$ групп $G_{1}, G$ в группе $S$ относительно почти гомоморфизма $J$ удовлетворяют следующим условиям: группа $\bar{G}_{1}(S)$ является нормальным делителем $в$ группе $\bar{G}(S)$, а факторгруппа $\bar{G}(S) / \bar{G}_{1}(S)$ коммутативна.

ДокАЗАТЕЛЬСтво. Нам надо доказать, что для каждой пары точек $a, b$ группы $\bar{G}(S)$ точка $a b a^{-1} b^{-1}$ принадлежит группе $\bar{G}_{1}(S)$, т. е. что в любой окрестности $V$ точки $a b a^{-1} b^{-1}$ существуют точки, принадлежащие образу $J\left(G_{1}\right)$ группы $G_{1}$. Согласно утверждению, примененному к слову $W=A_{1} A_{2} A_{1}^{-1} A_{2}^{-1}$, существуют окрестности $U_{1}$ и $U_{2}$ точек $a$ и $b$, такие, что для всякой пары точек $\tilde{a}, \tilde{b}$ группы $G$, для которых выполнены соотношения $J(\tilde{a}) \in U_{1}, J(\tilde{b}) \in U_{2}$, справедливо включение $J\left(\tilde{a} \tilde{b} \tilde{a}^{-1} \tilde{b}^{-1}\right) \in V$. Точки $a$ и $b$ лежат в группе $\bar{G}(S)$; поэтому точки $\tilde{a}$ и $\tilde{b}$ группы $G$, для которых эти соотношения выполнены, действительно существуют. Для такой пары точек $\tilde{a}, \tilde{b}$ точка $\tilde{a} \tilde{b} \tilde{a}^{-1} \tilde{b}^{-1}$ лежит в группе $G_{1}$, так как факторгруппа $G / G_{1}$ коммутативна. Итак, мы нашли точку, принадлежащую образу $J\left(G_{1}\right)$ группы $G_{1}$, в заданной окрестности $V$ точки $a b a^{-1} b^{-1}$. Теорема 1 доказана.

Теорема 2. Для всякого почти гомоморфизма $J: G \rightarrow T$ группы $G$ около группы $S$ и всякой подгруппы $G_{1}$ группь $G$, имеющей конечный индекс $k$ в группе $G$, индуцированные замыкания $\bar{G}_{1}(S), \bar{G}(S)$ групп $G_{1}, G$ в группе $S$ относительно почти гомоморфизма $J$ удовлетворяют следующему условию: группа $\bar{G}_{1}(S)$ является подгруппой конечного индекса в группе $\bar{G}(S)$, причем этот индекс меньше или равен $k$.

ДокАЗАТЕЛЬСтво. Пусть $R_{1}, \ldots, R_{k}$ - правые классы смежности группы $G$ по подгруппе $G_{1}$. Обозначим через $P_{i}, i=1, \ldots, k$, пересечение группы $S$ с замыканием образа $J\left(R_{i}\right)$ класса $R_{i}$ при отображении $J$. Мы покажем, что каждый правый класс смежности группы $\bar{G}(S)$ по группе $\bar{G}_{1}(S)$ совпадает с одним из множеств $P_{1}, \ldots, P_{k}$. Отсюда немедленно вытекает теорема 2 , так как это означает, что правых классов смежности не больше, чем $k$.

1) Покажем, что объединение множеств $P_{1}, \ldots, P_{k}$ совпадает с группой $\bar{G}(S)$. Действительно, группа $G$ является объединением правых классов смежности $R_{1}, \ldots, R_{k}$; поэтому $J(G)=\bigcup_{i=1}^{k} J\left(R_{i}\right)$. Отсюда следует, что $\bar{J}(G)=\bigcup_{i=1}^{k} \bar{J}\left(R_{i}\right)$. Пересекая множества, фигурирующие в этом равенстве, с группой $S$, получим $\bar{G}(S)=\bigcup_{i=1}^{k} P_{i}$.

2) Пусть $a$ - точка группы $\bar{G}(S)$ и $a \bar{G}_{1}(S)$ - правый класс смежности, содержащий эту точку. Согласно 1$)$, точка $a$ лежит в одном из множеств $P_{i}$. Покажем, что это множество $P_{i}$ содержит весь класс $a \bar{G}_{1}(S)$. Действительно, каждая точка этого класса имеет вид $b=a c$, где $c \in \bar{G}_{1}(S)$. Все три точки $a, b, c$ лежат в группе $S$. По определению почти гомоморфизма для каждой окрестности $V$ точки $b$ существуют окрестности $U_{1}$ и $U_{2}$ точек $a$ и $c$, такие, что если $J(\tilde{a}) \in U_{1}$ и $J(\tilde{c}) \in U_{2}$, то $J(\tilde{a} \tilde{c}) \in V$. Точки $\tilde{a}$ и $\tilde{c}$, удовлетворяющие этим соотношениям, можно выбрать соответственно в классе $R_{i}$ и группе $G_{1}$, так как $a \in \bar{J}\left(R_{i}\right), c \in \bar{G}_{1}(S)$. Для такого выбора точек $\tilde{a}$ и $\tilde{c}$ точка $\tilde{a} \tilde{c}$ лежит в классе $R_{i}$. Поэтому точка $b$ лежит в множестве $P_{i}$, что и требовалось доказать. 
3) Пусть множество $P_{i}$ непусто и $a \in P_{i}-$ одна из его точек. Покажем, что множество $P_{i}$ содержится в классе $a \bar{G}_{1}(S)$. Пусть $b-$ любая точка в $P_{i}$. Рассмотрим элемент $c=a^{-1} b$. Покажем, что $c \in \bar{G}_{1}(S)$. Для этого нужно показать, что всякая окрестность $V$ элемента $c$ пересекается с образом $J\left(G_{1}\right)$ группы $G_{1}$. Все три точки $a, b, c$ лежат в группе $S$. По определению почти гомоморфизма для каждой окрестности $V$ точки $c$ существуют окрестности $U_{1}$ и $U_{2}$ точек $a$ и $b$, такие, что если $J(\tilde{a}) \in U_{1}$ и $J(\tilde{b}) \in U_{2}$, то $J\left(\tilde{a}^{-1} \tilde{b}\right) \in V$. Точки $\tilde{a}, \tilde{b}$, удовлетворяющие этим соотношениям, можно выбрать в классе $R_{i}$, так как $a, b \in P_{i}$. Для такого выбора точек $\tilde{a}, \tilde{b}$ точка $\tilde{a}^{-1} \tilde{b}$ лежит в группе $G_{1}$. Поэтому точка $c$ лежит в группе $\bar{G}_{1}(S)$, что и требовалось доказать.

Теорема 2 доказана.

\section{§3. Индуцированное замыкание группы преобразований множества в группе преобразований его подмножества}

Опишем основной пример почти гомоморфизма $J$ около группы.

а) Топологическое пространство $T=T(L, X)$. Пусть $X-$ произвольное множество и $L \subseteq X-$ любое его подмножество. Рассмотрим пространство $T=T(L, X)$ отображений множества $L$ в множество $X$, наделенное следующей топологией. Для каждого отображения $f: L \rightarrow X$ и каждого конечного множества $K \subset L$ обозначим через $U_{K}(f)$ множество отображений из $L$ в $X$, совпадающих с отображением $f$ на множестве $K$. По определению базис окрестностей элемента $f$ в пространстве $T(L, X)$ образует совокупность множеств $\left\{U_{K}(f)\right\}$, где индекс $K$ пробегает множество всех конечных подмножеств в $L$. Другими словами, только что определенную топологию в множестве $T(L, X)$ можно описать как топологию поточечной сходимости отображений из $L$ в $X$ в дискретной топологии множества $X$. Для бесконечных множеств $X$ топология в $T(L, X)$ нетривиальна и полезна для дальнейшего.

b) Грynna $S=S(L) \subset T(L, X)$. Группа $S(L)$, состоящая из всех взаимно однозначных преобразований множества $L$, естественным образом вложена в пространство $T(L, X)$ : взаимно однозначное отображение $F: L \rightarrow L$ можно рассматривать и как отображение $f: L \rightarrow X$, так как $L \subset X$.

c) Группа $G$ и отображение $J(G) \rightarrow T(L, X)$. В качестве группы $G$ возьмем любую подгруппу группы $S(X)$ взаимно однозначных преобразований множества $X$. В качестве отображения $J: G \rightarrow T(L, X)$ рассмотрим отображение, сопоставляющее каждому отображению $f: X \rightarrow X$, принадлежащему группе $G$, его ограничение на множество $L$, т. е. $J(f)=\left.f\right|_{L}$.

Tеорема. B описанной выше в $n n . a), b)$, c) ситуации отображение $J: G \rightarrow$ $T(L, X)$ является почти гомоморфизмом около группы $S=S(L)$.

ДокАЗАТЕЛЬСтво. 1) Ограничение тождественного отображения множества $X$ на подмножество $L$ является тождественным отображением множества $L$. Поэтому $J$ переводит единицу группы $G$ в единицу группы $S(L)$.

2) Пусть $a \in S(L)$. Фиксируем произвольное конечное подмножество $K \subset L$ и рассмотрим окрестность $V=U_{K}\left(a^{-1}\right)$ элемента $a^{-1}$ в пространстве $T(L, X)$. Обозначим через $K_{1}$ образ множества $K$ при отображении $a: L \rightarrow L$. Пусть $\tilde{a}-$ преобразование из $S(X)$ и $J(\tilde{a})-$ его ограничение на $L, J(\tilde{a})=\left.\tilde{a}\right|_{L}$. Рассмотрим окрестность $U_{1}=U_{K_{1}}(a)$ элемента $a$. Если $J(\tilde{a}) \in U_{1}$, то $\left.J\left(\tilde{a}^{-1}\right)\right|_{K}=\left.a\right|_{K}$. 
3) Пусть $a, b \in S(L)$, и пусть $a b \in S(L)-$ их композиция $b \circ a$. Фиксируем произвольное конечное множество $K \subset L$ и рассмотрим окрестность $V=U_{K}(a b)$ элемента $a b$ в пространстве $T(L, X)$. Обозначим через $K_{1}$ образ множества $K$ при отображении $a: L \rightarrow L$. Пусть $\tilde{a}, \tilde{b}-$ преобразования из $S(X)$ и $J(\tilde{a}), J(\tilde{b})$ - их ограничения на множество $L, J(\tilde{a})=\left.\tilde{a}\right|_{L}, J(\tilde{b})=\left.\tilde{b}\right|_{L}$.

Рассмотрим окрестности $U_{1}=U_{K}(a)$ и $U_{2}=U_{K_{1}}(b)$ элементов $a$ и $b$. Если $J(\tilde{a}) \in U_{1}$ и $J(\tilde{b}) \in U_{2}$, то $J(\tilde{a} \tilde{b}) \in V$. Действительно, если $\left.\tilde{b}\right|_{K_{1}}=\left.b\right|_{K_{1}}$ и $\left.\tilde{a}\right|_{K}=\left.a\right|_{K}$, то $\left.\tilde{a} \tilde{b}\right|_{K}=\left.a b\right|_{K}$.

\section{§4. Группы монодромии индуцированных функций}

С каждой однозначной аналитической функцией $f$ можно связать ее струйное расширение $\mathscr{F}$, сопоставляющее каждой точке $x$ росток функции $f$ в этой точке. Аналогично можно говорить о струйном расширении $\mathscr{F}$ многозначной аналитической функции $f$ : это многозначная функция, принимающая значения в множестве ростков функции $f$ и сопоставляющая точке $x$ все регулярные ростки функции $f$ в этой точке.

Пусть $f$ - многозначная $\mathcal{S}$-функция на пространстве $\mathbb{C}^{n}$ и $f_{a}$ - некоторый выделенный росток функции $f$ в точке $a$. Непрерывное отображение $\pi:\left(Y, y_{0}\right) \rightarrow$ $\left(\mathbb{C}^{n}, a\right)$ линейно связного топологического пространства $Y$ с отмеченной точкой $y_{0}$ в пространство $\mathbb{C}^{n}$, такое, что $\pi\left(y_{0}\right)=a$, называется допустимым для ростка $f_{a}$, если росток $f_{a}$ аналитически продолжается вдоль образа любой кривой из пространства $Y$, начинающейся в отмеченной точке $y_{0}$.

ЗАмЕчАНИЕ. Типичный пример пространств $Y$, с которыми нам придется иметь дело, доставляют такие локально неодносвязные пространства, как дополнения комплексной прямой $\mathbb{C}$ до счетного всюду плотного множества $A \subset \mathbb{C}$, т.е. $Y=\mathbb{C} \backslash A$.

$\mathrm{C}$ допустимым для ростка $f_{a}$ отображением $\pi:\left(Y, y_{0}\right) \rightarrow\left(\mathbb{C}^{n}, a\right)$ свяжем многозначную функцию $\pi^{*} \mathscr{F}$ на пространстве $Y$, принимающую значения в множестве ростков многозначной функции $f$ в точках пространства $\mathbb{C}^{n}$. А именно, каждое значение многозначной функции $\pi^{*} \mathscr{F}$ в точке $y \in Y-$ это росток функции $f$ в точке $\pi(y) \in \mathbb{C}^{n}$, получающийся аналитическим продолжением ростка $f_{a}$ вдоль некоторой кривой вида $\pi \circ \gamma:[0,1] \rightarrow \mathbb{C}^{n}$, где $\gamma:[0,1] \rightarrow Y-$ кривая в пространстве $Y$, начинающаяся в точке $y_{0}$ и заканчивающаяся в точке $y$, т. е. $\gamma(0)=y_{0}, \gamma(1)=y$. Для многозначной функции $\pi^{*} \mathscr{F}$ на пространстве $Y$ определяется группа монодромии (которая вполне может оказаться континуальной) и монодромная пара ростка $f_{a}$ этой функции $\pi^{*} \mathscr{F}$ в точке $y_{0}$ точно так же, как это делалось для $\mathcal{S}$-функций.

Обозначим через $L_{a}$ совокупность всех ростков функции $f$ в точке $a$, являющихся значениями многозначной функции $\pi^{*} \mathscr{F}$ в точке $y_{0}$. Группа монодромии $M$ функции $\pi^{*} \mathscr{F}$ является транзитивной группой взаимно однозначных преобразований множества $L_{a}$. Нам нужно связать пару $M_{0}, M$, в которой $M_{0}-$ стационарная подгруппа ростка $f_{a}$ в группе $M$, с монодромной парой $\mathcal{S}$-функции $f$. Для этого нам понадобятся описываемые ниже отождествления.

Пусть $O-$ множество особых точек функции $f$ и $x \notin O-$ любая неособая точка в пространстве $\mathbb{C}^{n}$. Обозначим через $X$ множество всех ростков функции $f$ в точке $x$. Фиксируем кривую $\delta:[0,1] \rightarrow \mathbb{C}^{n}$, соединяющую точки $a$ и $x$, $\delta(0)=a, \delta(1)=x$, и пересекающую множество особенностей функции $f$ лишь, 
может быть, в начальный момент, т. е. $\delta(t) \notin O$ при $t>0$. Существование такой кривой $\delta$ вытекает из леммы о снятии кривой с тощего множества. Каждый из ростков функции $f$, лежащих в множестве $L_{a}$, аналитически продолжается вдоль кривой $\delta$. Действительно, ряд Тейлора каждого ростка из множества $L_{a}$ сходится в точках $\delta(t)$ кривой $\delta$ при достаточно малых $t, 0 \leqslant t \leqslant t_{0}$. При $t \geq t_{0}$ никаких препятствий к продолжению ростка не возникает, так как по условию при $t>0$ точки $\delta(t)$ не лежат в множестве $O$.

Отождествим каждый росток $f_{i a}$ из множества $L_{a}$ с ростком $f_{i x}$ в точке $x$, полученным при продолжении ростка $f_{i a}$ вдоль кривой $\delta$. При этом множество $L_{a}$ отождествится с некоторым подмножеством $L_{x}$ в множестве $X$, выделенный росток $f_{a}$ отождествится с некоторым выделенным ростком $f_{x} \in X$, группа монодромии $M$ функции $\pi^{*} \mathscr{F}$ отождествится с некоторой группой $M(x)$ транзитивных преобразований множества $L_{x}$, а стационарная подгруппа ростка $f_{a}$ в $M$ отождествится со стационарной подгруппой $M_{0}(x)$ ростка $f_{x}$ в группе $M(x)$. Обозначим через $G$ группу монодромии функции $f$, рассматриваемую как группа транзитивных взаимно однозначных преобразований множества $X$ в себя. Через $G_{0}$ обозначим стационарную подгруппу ростка $f_{x}$ в группе $G$.

Группы $G_{0}, G$ мы рассматриваем как группы преобразований множества $X$, содержащего подмножество $L_{x} \subset X$.

Теорема. Индуцзированное замькание $\bar{G}(S)$ группь монодромии $G$ функции $f$ в группе $S=S\left(L_{x}\right)$ взаимно однозначных преобразований множества $L_{x}$ содержит группу монодромии $M(x)$ функици $\pi^{*} \mathscr{F}$. При этом стационарная подгруппа $M_{0}(x)$ равна пересечению группь $M(x)$ с индуцированным замыканием $\bar{G}_{0}(S)$ стационарной подгруппь $G_{0}$ ростка $f_{x}$ в группе $G$.

ДокАЗАТЕЛЬСтво. Если росток аналитической функции аналитически продолжается вдоль некоторой кривой $\gamma:[0,1] \rightarrow \mathbb{C}^{n}$, то он аналитически продолжается вдоль всякой кривой $\tilde{\gamma}$ с теми же концами, $\gamma(0)=\tilde{\gamma}(0), \gamma(1)=\tilde{\gamma}(1)$, достаточно близкой к кривой $\gamma$. При этом продолжения этого ростка вдоль кривых $\gamma$ и $\tilde{\gamma}$ приводят к одному и тому же результату. Доказательство теоремы основано на этом аналитическом факте. Ввиду принятых отождествлений каждое преобразование $m: L_{x} \rightarrow L_{x}$ из группы $M(x)$ получается одновременным аналитическим продолжением ростков из множества $L_{x}$ вдоль некоторой кривой $\gamma$ вида $\delta \gamma_{1} \delta^{-1}$, где $\delta^{-1}-$ кривая $\delta$, проходящаяся в обратном направлении, а кривая $\gamma_{1}$ является образом при отображении $\pi$ некоторой кривой $\gamma_{2}:[0,1] \rightarrow Y$ в пространстве $Y$, начинающейся и заканчивающейся в точке $y_{0}$. Концы кривой $\gamma$ лежат в точке $x \in \mathbb{C}^{n}$, но она, вообще говоря, пересекает множество $O$ особых точек функции $f$. Фиксируем любое конечное множество $K$ ростков в $L_{x}$. Продеформируем немного кривую $\gamma$, оставляя закрепленными ее концы, таким образом, чтобы не изменить аналитические продолжения конечного множества $K$ ростков вдоль кривой, и так, чтобы продеформированная кривая $\tilde{\gamma}$ не пересекала множество $O$. Это можно сделать, учитывая аналитический факт, приведенный в начале доказательства, и лемму о снятии кривой с тощего множества.

Вдоль кривой $\tilde{\gamma}$ можно аналитически продолжать все ростки из множества $X$, так как она не пересекает множество $O$. Соответствующее кривой $\tilde{\gamma}$ преобразование $g: X \rightarrow X$ принадлежит группе монодромии $G$ функции $f$. Итак, мы для окрестности $U_{K}$ преобразования $m: L_{x} \rightarrow L_{x}$, лежащего в группе $M(x)$, предъявили преобразование $g: X \rightarrow X$ из группы $G$, такое, что $\left.m\right|_{K}=\left.g\right|_{K}$. Поэтому $M(x) \subset \bar{G}(S)$. 
Далее, подгруппа $M_{0}(x)$ состоит из преобразований группы $M(x)$, переводящих точку $f_{x}$ в себя. Для конечных множеств $K \subset L_{x}$, содержащих точку $f_{x}$, всякое преобразование $g: X \rightarrow X$, совпадающее на множестве $K$ с некоторым преобразованием $m: L \rightarrow L$, где $m \in M_{0}$, тоже переводит точку $f_{x}$ в себя. Поэтому $M_{0}=M \cap \bar{G}_{0}(S)$.

Теорема доказана.

\section{§5. Классы пар групп}

В одномерном топологическом варианте теории Галуа описывается, как изменяются монодромные пары функций одной переменной при суперпозициях, интегрированиях, дифференцированиях и т. д. Для этого используются некоторые понятия, связанные с парами групп. Для функций многих переменных в связи с теоремой из $\$ 4$ эти понятия необходимо несколько модифицировать. Напомним определения и проведем нужную модификацию.

Парой грyпn мы всегда называем пару, состоящую из группы и некоторой ее подгруппы, при этом группа отождествляется с парой групп, состоящей из этой группь и ее единичной подгруппь.

ОПреДЕЛЕНИЕ. Совокупность $\mathscr{L}$ пар называется почти полным классом пар групn, если для каждой пары групп $\left[\Gamma, \Gamma_{0}\right] \in \mathscr{L}$, где $\Gamma_{0} \subseteq \Gamma$, выполняются следующие условия:

1) для любого гомоморфизма $\tau: \Gamma \rightarrow G$, где $G-$ некоторая группа, пара групп $\left[\tau \Gamma, \tau \Gamma_{0}\right]$ также содержится в $\mathscr{L}$,

2) для любого гомоморфизма $\tau: G \rightarrow \Gamma$, где $G-$ некоторая группа, пара групп $\left[\tau^{-1} \Gamma, \tau^{-1} \Gamma_{0}\right]$ также содержится в $\mathscr{L}$,

$3)$ для любой группы $G$, наделенной $T_{2}$-топологией и содержащей группу $\Gamma$, $\Gamma \subseteq G$, пара групп $\left[\bar{\Gamma}, \bar{\Gamma}_{0}\right]$ также содержится в $\mathscr{L}$, где $\bar{\Gamma}, \bar{\Gamma}_{0}-$ замыкания групп $\Gamma, \Gamma_{0}$ в группе $G$.

ОПРеДЕЛЕНИЕ. Почти полный класс пар групп $\mathscr{M}$ называется полныı, если

1) для каждой пары групп $\left[\Gamma, \Gamma_{0}\right] \in \mathscr{M}$ и группы $\Gamma_{1}$, такой, что $\Gamma_{0} \subseteq \Gamma_{1} \subseteq \Gamma$, пара групп $\left[\Gamma, \Gamma_{1}\right]$ также содержится в $\mathscr{M}$,

2 ) для каждых двух пар групп $\left[\Gamma, \Gamma_{1}\right],\left[\Gamma_{1}, \Gamma_{2}\right] \in \mathscr{M}$, таких, что $\Gamma_{2} \subseteq \Gamma_{1} \subseteq \Gamma$, пара групп $\left[\Gamma, \Gamma_{2}\right]$ также содержится в $\mathscr{M}$.

Минимальные почти полный и полный классы пар групп, содержащие фиксированное множество пар групп $\mathscr{B}$, будем обозначать соответственно через $\mathscr{L}\langle\mathscr{B}\rangle$ и $\mathscr{M}\langle\mathscr{B}\rangle$. Пусть $\mathscr{K}-$ класс всех конечных групп, $\mathscr{A}-$ класс всех абелевых групп, $S(k)$ - группа всех перестановок $k$ элементов. Минимальные полные классы пар групп $\mathscr{M}\langle\mathscr{A}, \mathscr{K}\rangle, \mathscr{M}\langle\mathscr{A}, S(k)\rangle$ и $\mathscr{M}\langle\mathscr{A}\rangle$ называются соответственно почти разрешимыми, $k$-разрешимьмми и разрешимыми классами пар групп. Эти классы пар групп важны в теории Галуа. Они допускают следующее явное описание.

Цепочка подгрупп $\Gamma_{i}, i=1, \ldots, m, \Gamma=\Gamma_{1} \supseteq \cdots \supseteq \Gamma_{m} \subseteq \Gamma_{0}$, называется нормальной башней пары групп $\left[\Gamma, \Gamma_{0}\right]$, если группа $\Gamma_{i+1}$ является нормальным делителем группы $\Gamma_{i}$ при каждом $i=1, \ldots, m-1$. Совокупность факторгрупп $\Gamma_{i} / \Gamma_{i+1}$ называется совокупностью делителей относительно нормальной башни.

ТЕОРЕМА О КЛАССАХ ПАР $\mathscr{M}\langle\mathscr{A}, \mathscr{K}\rangle, \mathscr{M}\langle\mathscr{A}, S(n)\rangle$ и $\mathscr{M}\langle\mathscr{A}\rangle[2,3]$. (1) Пара гpynn почти разрешима, если и только если она обладает такой нормальной башней, ито каждьй делитель относительно этой башни является или конечной, или коммутативной группой. 
(2) Пара групп k-разрешима, если и только если она обладает такой нормальной башней, что каждый делитель относительно этой башни является или подгруппой группы $S(k)$, или коммутативной группой.

(3) Пара групп разрешима, если и только если группа монодромии этой паpы paзрешuма (группой монодромии пары групп $\left[\Gamma, \Gamma_{0}\right]$ называется факторгруппа группы $\Gamma$ по наибольшему нормальному делителю, лежащему в группе $\Gamma_{0}$ ).

Усилим свойство 3) из определения почти полного класса пар групп:

$\left.3^{\prime}\right)$ для всякого почти гомоморфизма $J: \Gamma \rightarrow T$ около группы $S$ пара групп $\left[\bar{\Gamma}(S), \bar{\Gamma}_{0}(S)\right]$, где $\bar{\Gamma}(S), \bar{\Gamma}_{0}(S)-$ индуцированные замыкания групп $\Gamma, \Gamma_{0}$ в группе $S$ при почти гомоморфизме $J$, также содержится в $\mathscr{L}$.

ОПРЕДЕЛЕНИЕ. I-nочти полный Класс пар груnn, I-nолный класc nap груnn и классы $I \mathscr{L}\langle\mathscr{B}\rangle, I \mathscr{M}\langle\mathscr{B}\rangle$ определяются так же, как соответственно почти полный класс пар групп, полный класс пар групп и классы $\mathscr{L}\langle\mathscr{B}\rangle, \mathscr{M}\langle\mathscr{B}\rangle$, нужно лишь во всех определениях свойство 3) заменить более сильным свойством $3^{\prime}$ ).

ПреДЛОЖениЕ. Справедливы равенства I $\mathscr{M}\langle\mathscr{A}, \mathscr{K}\rangle=\mathscr{M}\langle\mathscr{A}, \mathscr{K}\rangle, I \mathscr{M}\langle\mathscr{A}, S(k)\rangle$ $=\mathscr{M}\langle\mathscr{A}, S(k)\rangle$ u $I \mathscr{M}\langle\mathscr{A}\rangle=\mathscr{M}\langle\mathscr{A}\rangle$.

ДокАЗАТЕЛЬСтво немедленно вытекает из явного описания классов $\mathscr{M}\langle\mathscr{A}, \mathscr{K}\rangle$, $\mathscr{M}\langle\mathscr{A}, S(k)\rangle$ и $\mathscr{M}\langle\mathscr{A}\rangle$ и из теорем 1 и $2 \$ 2$ об индуцированных замыканиях.

Теорема. Монодромная пара всякой многозначной функции, индуцированной при некотором непрерывном отображении S-функцией $f$, принадлежит минимальному I-почти полному классу пар групп, содержащему монодромную пару функции $f$. В частности, если группа монодромии $\mathcal{S}$-функции $f$ разрешима (монодромная пара почти разрешима, $k$-разрешима), то этим же свойством обладает группа монодромии (монодромная пара) всякой многозначной функции, индуцированной при некотором непрерывном отображении функцииеи $f$.

ДокАЗАТЕЛЬСтво вытекает из теоремы $\$ 4$ и из замкнутости классов $\mathscr{M}\langle\mathscr{A}, \mathscr{K}\rangle$, $\mathscr{M}\langle\mathscr{A}, S(k)\rangle$ и $\mathscr{M}\langle\mathscr{A}\rangle$ относительно операции индуцированного замыкания.

\section{ЛИТЕРАТУРА}

1. Хованский $A$. О представимости функций в квадратурах. УМН, 26, вып. 4, 251-252 (1971).

2. Хованский А. О представимости функций в квадратурах. Дисс. к.ф.-м.н., М., МИАН, M., 1973.

3. Khovanskii A. Topological obstructions for representability of functions by quadratures. J. Dynamical and Control Systems, 1, No. 1, 99-132 (1995).

4. Хованский $A$. О продолжаемости многозначных аналитических функций на аналитическое подмножество. Функц. анализ и его прил., 35, вып. 1, 62-73 (2001).

5. Khovanskii A. A multi-dimensional topological version of Galois theory. In: Proceeding of International Conference "Monodromy in Geometry and Differential Equations," 25-30 June, Moscow, 2001.

6. Хованский $A$. О суперпозициях голоморфных функций с радикалами. УМН, 26, вып. 2, 213-214 (1971).

7. Алексеев В. Б. Теорема Абеля в задачах и решениях. МЦНМО, М., 2001.

8. Арнольд В. И., Петровский И. Г. Топологические проблемы Гильберта и современная математика. УМН, 57, вып. 4, 197-207 (2002). 